\title{
Axon deviation in the human lamina cribrosa
}

\author{
James E Morgan, Glen Jeffery, Alexander J E Foss
}

\begin{abstract}
Aims-To examine the course taken by individual retinal ganglion cell axons through the human lamina cribrosa. Methods-Retinal ganglion cell axons were labelled using the retrograde tracer horseradish peroxidase applied directly to the optic nerve in two normal human eyes removed during the course of treatment for extraocular disease.

Results-A majority of axons took a direct course through the lamina cribrosa but a significant minority, in the range $8-12 \%$, deviated to pass between the cribrosal plates in both central and peripheral parts of the optic disc.

Conclusions-It is postulated that these axons would be selectively vulnerable to compression of the lamina cribrosa in diseases such as glaucoma in which the intraocular pressure is increased.

(Br F Ophthalmol 1998;82:680-683)
\end{abstract}

In the human eye the lamina cribrosa consists of a series of collagenous plates with several hundred perforations through which retinal ganglion cell axons pass to enter the optic nerve. The course taken by these axons is central to our understanding of diseases such as glaucoma in which raised intraocular pressure is associated with distortion of the lamina cribrosa $^{1}$ and subsequent axon compression. Axoplasmic transport may be disrupted ${ }^{2}$ thereby predisposing to retinal ganglion cell death. ${ }^{3}$

Detailed knowledge of the axon paths through the lamina cribrosa is essential if we are to understand the mechanisms by which axon compression may occur. Within the lamina, axon bundles appear to follow a relatively direct course as they pass through successive pores ${ }^{1}$ running with their neighbours towards the optic nerve head to be grouped in a retinotopic arrangement behind the eye. ${ }^{4}$ The course taken by individual axons within these bundles remains to be defined. Evidence from other systems suggests that this may not be entirely direct. For example, axons can deviate from their expected topographic pattern in both the vertical and horizontal aspects of the nerve fibre layer ${ }^{5}$ and the optic nerve. ${ }^{6}$ Indeed, some axon deviation might be expected since the number of cribrosal pores increases in the more posterior layers, resulting in the division of fascicular bundles. ${ }^{7}$ Such deviations might result in retinal ganglion cell axons passing between the plates of the cribrosa, rendering them vulnerable to the compressive effects of increased intraocular pressure.

In order to investigate this possibility, the course of axons through the lamina cribrosa of the human optic nerve head was traced in two human eyes obtained from mature male subjects following exenteration for extraocular orbital disease which had not compromised the eye or the optic nerve head. Axons were labelled using horseradish peroxidase (HRP) transported retrogradely from injections made in the cut end of the optic nerve.

\section{Methods}

Neuroanatomical investigations were carried out on two eyes removed from adult males (ages 44 and 86 years) and conformed to the ethical standards requirements of Moorfields Eye Hospital, London. Both eyes were removed in the course of treatment for advanced malignant neoplasia of the ocular adnexa. In neither case had tumour growth compromised the optic nerve or undergone intraocular extension. Intraocular pressures as measured by Goldmann tonometry just before surgery, were within the normal range (less than $21 \mathrm{~mm}$ $\mathrm{Hg}$ ).

Each globe was rapidly removed following transection of the optic nerve. The remaining 1-2 cm stump of optic nerve was then carefully trimmed under microscopic observation to 1-2 $\mathrm{mm}$. The anterior segment was excised and the globe placed in a solution consisting of $0.21 \mathrm{~g} / 1$ sodium bicarbonate, $0.18 \mathrm{~g} / 1$ sucrose, and $0.89 \%$ Ames medium (Sigma), saturated with a mixture of $95 \%$ oxygen and $5 \%$ carbon dioxide. Cuts in the retina were used to preserve ocular orientation. The vitreous was gently removed and a glass micropipette tip (outside diameter $100 \mu \mathrm{m}$ ) coated with HRP (Sigma type VI, $70 \%$ solution in distilled water) placed into the superior and inferior quadrants of the sectioned end of the optic nerve. A single penetration was made at each site. The pipette was left in place for approximately 5 minutes to allow diffusion of the HRP into the optic nerve and the globe returned to the incubation medium between injections.

The globes were incubated for approximately 12 hours at $37^{\circ} \mathrm{C}$ in the gassed medium. The retinae were then fixed by
Accepted for publication 9 January 1998 
immersion in $2 \%$ paraformaldehyde and $2 \%$ glutaraldehyde in $0.1 \mathrm{M}$ phosphate buffer $(\mathrm{pH}$ 7.4 ), placed in $25 \%$ sucrose in phosphate buffer overnight, embedded in gelatin and cut as frozen sections at $60 \mu \mathrm{m}$. Sections were reacted for HRP using DAB as the chromogen, ${ }^{8}$ mounted on subbed slides, dehydrated, cleared, and stained with cresyl violet.

\section{Results}

High quality retrograde axonal labelling was achieved in both optic nerve heads. Figure 1 summarises the histological findings from one eye; similar axon trajectories were found in the other eye. Axons could be traced from the retrolaminar part of the optic nerve, through the lamina cribrosa and, in many cases, several hundred micrometres into the peripapillary retinal nerve fibre layer. The plates of the lamina cribrosa were easily discerned in unstained sections as zones that formed a regular meshwork devoid of blood vessels. The location of these zones was confirmed with the Nissl counterstain. The majority of axons passed through the lamina cribrosa with little deviation. However, a number were seen to pass around and between the plates of the lamina cribrosa before entering the retrolaminar region of the optic nerve.

The line diagram of the optic nerve head in Figure 1 shows the areas that are outlined in greater detail. Shaded areas correspond to the plates of the lamina cribrosa. Blood vessels (BV) could easily be identified by the presence within their lumen of red blood cells. In Figure 1A axons course fairly directly through the
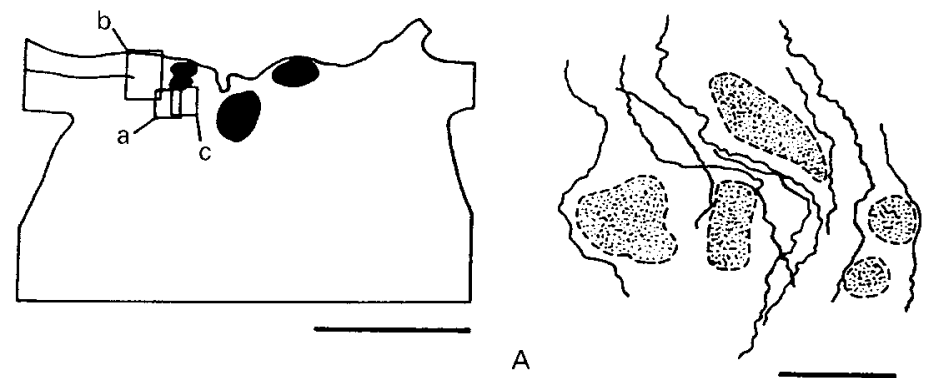

\section{A}

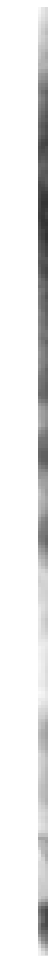

Figure 1 Camera lucida drawing showing the path taken by individual HRP labelled axons through the optic nerve head. Transverse section. Optic nerve showing the location of sample areas $a, b$, and $c$ Scale bar $1 \mathrm{~mm} . A, B$, and $C$ are areas in the region of the lamina cribrosa drawn at higher magnification. Stippled areas show regions corresponding to the location of the cribrosal plates. $B V=$ blood vessel (filled areas in all figures). Scale bar $50 \mu \mathrm{m}$.

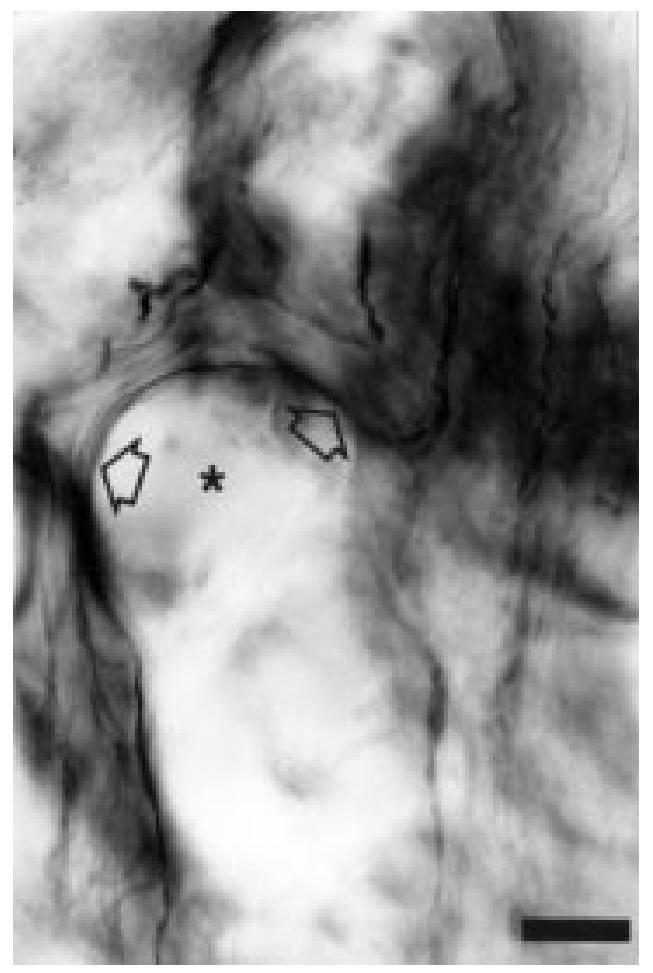

Figure 2 Photomicrograph from the optic nerve head showing deviation of HRP labelled axons in the vicinity of a cribrosal plate (asterisk). Arrows highlight the axon of interest. The top of the image represents more anterior optic nerve. Scale bar $2 \mu \mathrm{m}$.

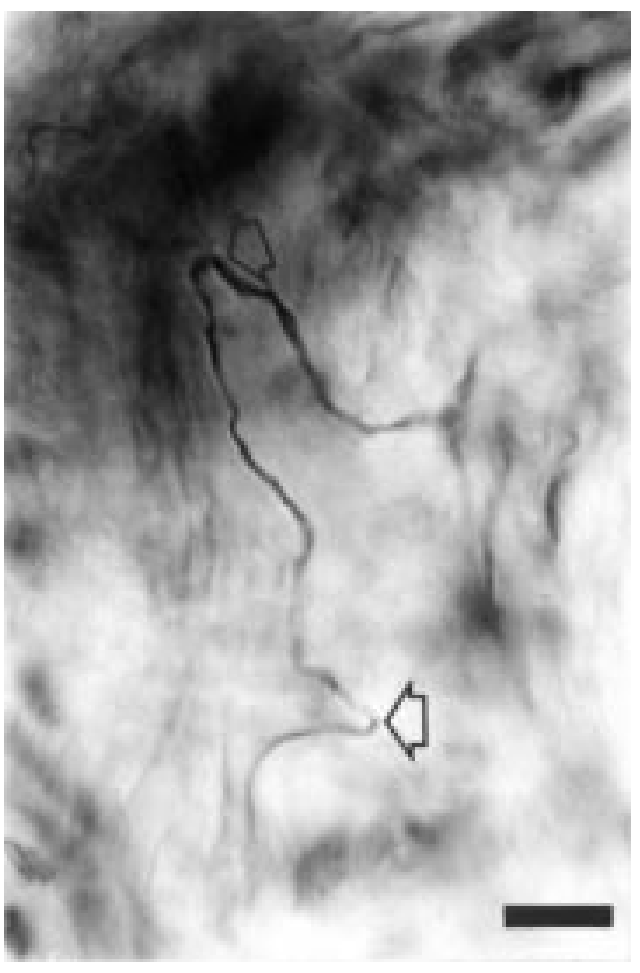

Figure 3 Photomicrograph of single deviating axon in the vicinity of a cribrosal plate taken from the optic nerve in Figure 1. The top of the image represents more anterior optic nerve. Arrows highlight deflections in the axon of interest. Scale bar $2 \mu \mathrm{m}$. 
lamina cribrosa. However, as many as half of the axons in this field of view showed some deviation, running between the plates of the lamina cribrosa. Various axon trajectories are illustrated in Figure 1B in which three axons pass with little deviation while three others show marked deviation, crossing the path of the first group. Indeed, one of the axons undergoes two $180^{\circ}$ changes in direction as it passes between cribrosal pores. Figure 1C shows axons from an area of heavy labelling that are representative of the course taken by some axon bundles. All the axons in this case underwent marked deviation, passing between the plates of the lamina cribrosa. In the examples shown, labelled axons were only drawn from a single section and have not been followed between sections.

Figure 2 shows a photomicrograph from the superior pole of same optic nerve head showing an example of axon deviation (arrow) over a beam in the lamina cribrosa (asterisk), before passing through the next pore. To the right of this, several axons can be seen taking a more direct route through the cribrosa.

An example of marked axon deviation is shown in Figure 3 as an axon deviates through more than $180^{\circ}$ before passing forward in the optic nerve head. The points of inflection are marked by arrows. Other axons that are slightly out of the plane of focus can be seen to run close to this axon but take a direct route through the lamina cribrosa.

Estimation of the proportion of axons that followed an indirect pathway through the cribrosa is difficult in those areas with heavy axon labelling. In areas of lighter labelling where all the axons in a field could be identified, the proportion of axons with indirect pathways could be determined. Counts were made in central and peripheral regions of both nerve heads; $12 \%$ of 465 axons examined in the first nerve and $8 \%$ of 231 axons examined in the second nerve underwent deviation as described in Figures 1-3.

\section{Discussion}

To our knowledge, this is the first study to detail the course taken by individual axons through the human lamina cribrosa. We describe a population of retinal ganglion cell axons that do not take the most direct path through the lamina cribrosa but run instead between the cribrosal plates as they pass through cribrosal pores to enter the retrolaminar region of the optic nerve. An important technical consideration is that the observed deviation may have resulted from tissue distortion during histological processing. We think that this is unlikely for several reasons. Axon misdirection could be seen before dehydration and, following dehydration, it was possible to observe both straight and deviated axons passing through the same cribrosal pore (Fig 1B). It is also unlikely that the deviation reflects trauma induced during surgical removal of the eye. The initial (surgical) transection sites were at least $1 \mathrm{~cm}$ from the HRP injection sites and final trimming of the optic nerve stump was made under micro- scopic observation with minimal tissue distortion. Furthermore, while the process of transection may conceivably have resulted in local axon distortion, it is difficult to see how this could explain the interlaminar trajectory of some axons (Fig 1A).

Our findings are consistent with a recent study $^{9}$ that calculated the ratio of the length of the axon channel through the cribrosa with respect to cribrosal thickness in the human optic nerve head. The ratio varied in both central and peripheral parts of the nerve, ranging from 1.0 (indicating a straight passage) to 1.46 (indicating a more convoluted passage), as would be expected from a mixed population of deviating and non-deviating axons (Fig 2). Since compaction of the lamina cribrosa has been described in histological studies of glaucomatous eyes ${ }^{1}$ one implication of our findings is that these interlaminar axons, which were found in both central and peripheral parts of the optic nerve will be particularly vulnerable to the compressive effects of raised intraocular pressure. Such damage would be in addition to any shearing effect that would occur in eyes with advanced glaucomatous damage where peripheral regions of the lamina cribrosa rotate with respect to the axon stream and are displaced both posteriorly and peripherally. ${ }^{10}$ In central parts of the cribrosa this rotation would be less marked but deviating axons would still be vulnerable to compression. In view of the considerable evidence that larger axons are selectively damaged in glaucoma, ${ }^{11}{ }^{12}$ it would be important to know whether the deviating axons have axon diameters larger than the mean for the optic nerve. In the present material, we were unable to measure directly the diameters of the deviating axons. However, such axons were found throughout the nerve and did not cluster in the periphery, where the larger axons from more peripheral retinal ganglion cells might be found. ${ }^{413}$

The simplest explanation for the axon deviation is that it is the result of topographic imprecision in the retinogeniculate pathway as seen in other species. ${ }^{6}$ Alternatively, it is possible that it represents an attempt by axons to achieve the correct topographic location in the retrolaminar part of the optic nerve. In the primate (macaque) retinal nerve fibre layer, the axons of peripheral retinal ganglion cells run anterior (superficial) to those arising from more central retinal ganglion cells. ${ }^{5}$ Since these axons lie more peripheral in the optic nerve ${ }^{4}$ some rearrangement would be needed to match these topographic patterns. Studies of axon paths in prelaminar optic nerve provide evidence that this occurs. ${ }^{5}$ In the present study this process may have been obscured because of the density of axon labelling. In addition, in the human, axon rearrangement may be constrained by the tight organisation of astrocytes in the prelaminar region of the optic nerve. ${ }^{14}$ By contrast, in the lamina itself , astrocytes are relatively sparse in the regions between the cribrosal plates, ${ }^{14}$ so allowing for some axon deviation. 
Our findings add support to the concept that mechanical axonal compression may be a factor in diseases such as glaucoma. The cause of axon misdirection remains unclear and raises the need for further studies of axon trajectory in the lamina cribrosa.

JEM was supported in part by the International Glaucoma

We are grateful for the assistance of Phil Luthert and to Dave West for advice on the incubation medium.

1 Quigley H, Addicks E, Green W, et al. Optic nerve damage in human glaucoma. II. The site of injury and susceptibility to damage. Arch Ophthalmol 1981;99:635-49.

2 Quigley H, Addicks E. Chronic experimental glaucoma in primates. II. Effect of extended intraocular pressure on optic nerve head and axonal transport. Invest Ophthalmol Vis Sci 1980;19:137.

3 Quigley H, Nickells R, Kerrigan L, et al. Retinal ganglion cell death in experimental glaucoma and after axotomy occurs by apoptosis. Invest Ophthalmol Vis Sci 1995;36: 774-86.

4 Hoyt W, Luis O. Visual fiber anatomy in the infrageniculate pathway of the primate. Arch Ophthalmol 1962;111:645-
5 Ogden T. Nerve fiber layer of the macaque retina: retinotopic organization. Invest Ophthalmol Vis Sci 1983;24:85-98.

6 Horton J, Greenwood M, Hubel D. Non-retinotopic arrangement of fibres in cat optic nerve. Nature 1979;282: $720-2$

7 Ogden T, Duggan J, Danley K, et al. Morphometry of nerve bundle pores in the optic nerve head of the human. Exp Eye Res 1988;46:559-68.

8 Mesulam M-M, Rosene D. Sensitivity in horseradish peroxidase neurochemistry: a comparative and quantitative analysis of nine methods. 7 Histochem Cytochem 1979:27: 763-73.

9 Dichtl A, Jonas J, Naumann G. Course of the optic nerve fibers through the lamina cribrosa in human eyes. Graefes Arch Clin Exp Ophthalmol 1996;234:581-5.

10 Yan D, Coloma F, Metheetrairut A, et al. Deformation of the lamina cribrosa by elevated intraocular pressure. $\mathrm{Br} F \mathrm{Oph}$ thalmol 1994;78:643-8.

11 Quigley H, Sanchez R, Dunkelberger G, et al. Chronic glaucoma selectively damages large optic nerve fibers. Invest Ophthalmol Vis Sci 1987;28:913-20.

12 Quigley HA. Chronic human glaucoma causing selectively greater loss of large optic nerve fibers. Ophthalmology 1988; 95:357-63.

13 Ogden T. Nerve fiber layer of the primate retina: morphometric analysis. Invest Ophthalmol Vis Sci 1984;25: $19-29$.

14 Trivino A, Ramirez J, Salazar J, et al. Immunohistochemical study of human optic nerve head astroglia. Vis Res study of human 